\title{
Ageusia: A Symptom of Peritonsillar Abscess?
}

\author{
K. Revathishree ${ }^{1}$ (D) S. Shyam Sudhakar ${ }^{1}$
}

Received: 16 December 2020/ Accepted: 2 March 2021 / Published online: 10 March 2021

(C) Association of Otolaryngologists of India 2021

\begin{abstract}
Peritonsillar abscess is a known complication of tonsillitis. The patient usually presents with typical symptoms of odynophagia, fever and difficulty in mouth opening. The diagnosis is established by clinical examination that commonly revealed unilateral peritonsillar swelling. Aspiration of pus will confirm the diagnosis. We report an atypical presentation of peritonsillar abscess case which presented only with loss of taste sensation without dysphagia, fever, odynophagia or trismus.
\end{abstract}

Keywords Ageusia P Peritonsillar abscess .

Chorda tympani nerve $\cdot$ Taste $\cdot$ Tonsillitis

\section{Introduction}

Peritonsillar abscess is a relatively common condition, occurring in about 30 persons per 100,000 per year and thus accounting for approximately 45,000 cases per year [1]. Quinsy or peritonsillar abscess is the collection of pus within the peritonsillar space [2]. The typical clinical presentations include odynophagia, dysphagia, drooling of saliva, muffled voice and high grade fever. Trismus is a pathognomic sign of a patient with peritonsillar infection. These symptoms depend on the severity and size of the abscess [2]. We present a case of peritonsillar abscess with loss of taste with no other symptom.

\section{K. Revathishree}

revathishree23@gmail.com

1 Department of ENT-HNS, Saveetha Medical College and Hospital, No. 1, Saveetha Nagar, Thandalam, Chennai 602105, Tamilnadu, India

\section{Case Summary}

A 36 year old gentleman presented to ENT outpatient department with complaints of loss of taste sensation for the past 2 weeks and incidentally noticed a swelling in the oral cavity. There was no associated history of throat pain, fever, dysphagia, odynophagia, trismus, breathlessness, drooling of saliva, foreign body sensation and change in voice. There was no history of recurrent tonsllititis or peritonsillar abscess in the past. He is a known smoker for past 5 years and smokes $4-5$ cigarettes/day. There was no other medical illness like diabetes mellitus and hypertension. Patient has not taken any treatment for above complaint. No history of loss of weight or loss of appetite was elicited. He was not on any chronic medications/native treatment.

On examination of oral cavity, mouth opening was adequate (Fig. 1), right tonsil medialised, pushing uvula and soft palate to left (Fig. 2). On palpation, mass is fluctuant and non tender. Ballotability noted. Examination of opposite side tonsil was normal. No other active septic foci noted in the throat. No sinus / mastoid tenderness noted. Laryngeal crepitus was present. On examination of neck level IIb lymph nodes were palpable and non tender. No other neck swelling / sinuses / scars noted. Other system examination was normal. Covid-19 RT-PCR was negative. On routine blood examination, total count was mildly increased. CECT neck was taken which revealed an abscess collection in the right peritonsillar space (Fig. 3). Ultrasonogram of neck was planned but deferred as loss of taste was present and deeper pathologies if any could not be fully visualized due to bony confinements in the head and neck area.

Taste sensation was tested using four different solutions for sweet, salt, sour and bitter with sucrose (10\%).sodium 


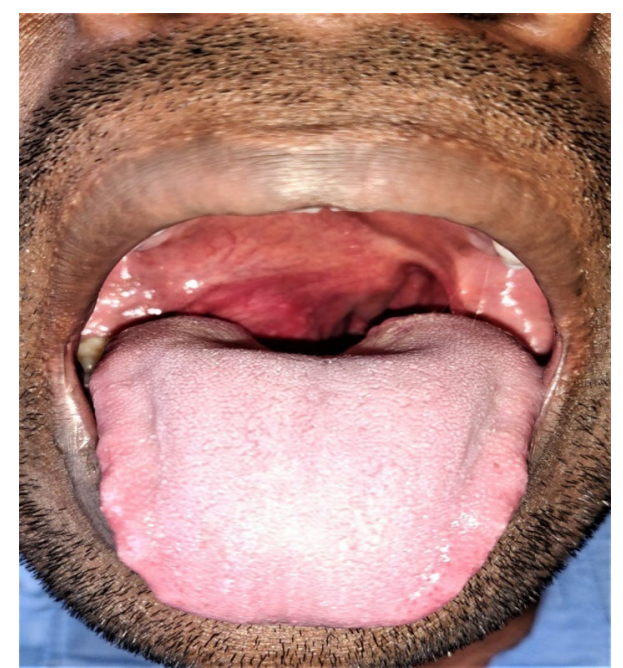

Fig. 1 Adequate mouth opening

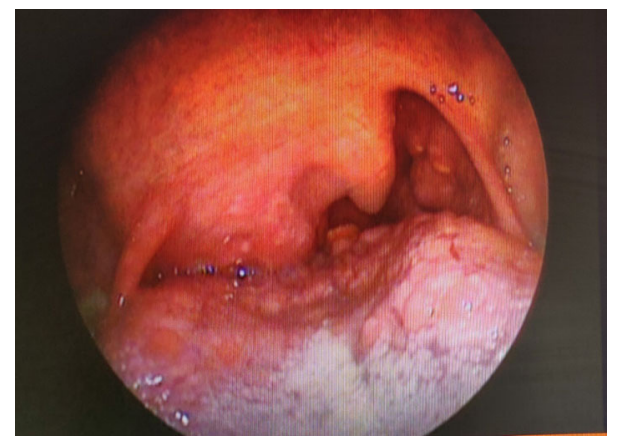

Fig. 2 Right peritonsillar abscess pushing uvula to opposite side

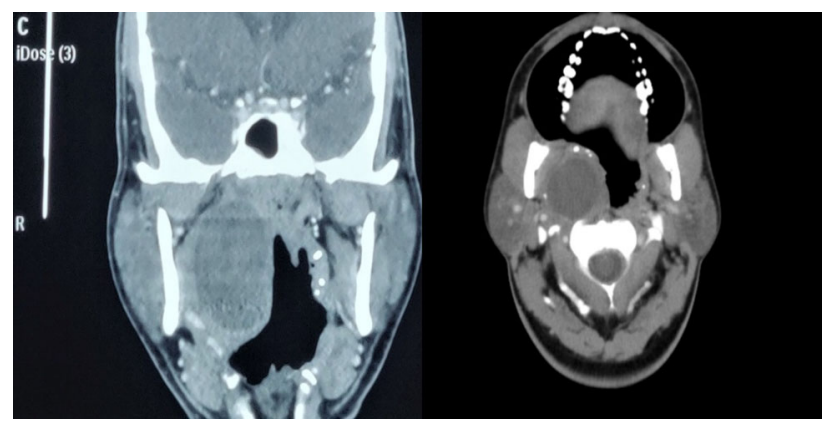

Fig. 3 CT Neck showing right peritonsillar abscess

chloride (15\%), acetic acid (1\%) and quinine sulphate respectively. Patient had no taste sensation in the right anterior $2 / 3$ rd of tongue. Aspiration was attempted and thick pus was collected and sent for culture and sensitivity. Incision \& Drainage was done and pus drained, patient was treated with a course of antibiotic and discharged. His taste sensation was tested 1 week after discharge and patient was able to identify all tastes.

\section{Discussion}

Peritonsillar abscess is defined as the collection of purulent secretion between the fibrous capsule of the palatine tonsil and the pharyngeal superior constrictor muscle [2]. It is a potential space which contains loose connective tissue. If an infection occurs, there will be a period of peritonsillar cellulitis without abscess followed by abscess formation [1].

A review on pathogenesis of peritonsillar abscess reports that peritonsillar abscess is not necessarily an extension of acute exudative tonsillitis but abscess formation of weber's salivary glands in the supratonsillar fossa [3]. Additional factors such as smoking and periodontal disease may also contribute to the formation of a peritonsillar abscess [4].

The common symptoms include severe sore throat pain associated with high grade fever, malaise, dysphagia, odynophagia and ipsilateral otalgia due to referred pain. Drooling or pooling of saliva is often present due to severe pain associated with swallowing [5].

In another study by Galioto et al., most common Symptoms include fever, sore throat, dysphagia, trismus, and a "hot potato" voice [4] where as in our case patient did not present with any of above symptoms.

Taste sensation was tested using four different solutions for sweet, salt, sour and bitter with sucrose (10\%), sodium chloride (15\%), acetic acid (1\%) and quinine sulphate. Patient was asked to rinse his mouth before testing with each solution and each side of the tongue was tested separately, and anterior and posterior part of tongue tested separately without closing the mouth after application of each solution using a cotton swab. Oral cavity is cleansed with water before testing each solution and dried with gauze. Patient was given a card with all four tastes and asked to tick in the card response was received as Intense $(++++)$, Moderate $(+++)$, mild $(++)$, slight $(+)$, absent (0) [6].

In this case patient only presented with loss of taste which is an unusual presentation of peritonsillar abscess and patient had absent taste sensation for all 4 tastes in the right anterior $2 / 3$ rd of tongue and after incision and drainage taste sensation score was interpreted as moderate $(+++)$, which shows improvement in taste perception.

The chorda tympani nerve joins the lingual nerve lower in the infratemporal fossa, approximately one centimeter below the bifurcation of the lingual and inferior alveolar nerves, after it exits the base of the skull via the pterygotympanic fissure. Once the LN enters the pterygomandibular space, its traverses between the lateral aspect of the medial pterygoid muscle and medial aspect of the mandibular ramus $[7,8]$. 
Computed tomography scan of the neck is done in peritonsillar abscess which is done to distinguish between peritonsillar cellulitis and peritonsillar abscess, as well to identify the spread of the infection to deep neck spaces [9]. In treatment of peritonsillar abscess incision and drainage with accompanying antimicrobial therapy and hydration are the cornerstones of management [10].

In our case loss of taste sensation is due to probable involvement of lingual nerve in pterygomandibular space due to mechanical compression, which improved after treatment.

\section{Conclusion}

Although peritonsillar abscess is a common condition, ageusia is not a common presentation in the absence of other symptoms. A probable hypothesis is that mechanical compression of lingual nerve in pterygomandibular space can lead to loss of taste. This is vital information to not get misled while diagnosing patients with ageusia in the midst of an ongoing pandemic.

Funding No external funding.

\section{Declaration}

Conflict of interest The authors declare that they have no conflict of interest.
Ethics Approval Institutional Ethical Committee approval obtained.

\section{References}

1. Herzon FS (1995) Peritonsillar abscess: incidence, current management practices, and a proposal for treatment guidelines. Laryngoscope 105(Suppl 74):1-17

2. Yaroko AA, Irfan M (2011) Atypical presentation of peritonsillar abscess. Bangladesh J Med Sci 10(4):293-295

3. Passy V (1994) Pathogenesis of peritonsillar abscess. Laryngoscope 104(2):185-190

4. Nwe TT, Singh B (2000) Management of pain in peritonsillar abscess. J Laryngol Otol 114(10):765-767

5. Galioto NJ (2017) Peritonsillar abscess. Am Fam Physician 95(8):501-506

6. Ghai CL (1999) A textbook of practical physiology 8 th. Jaypee Brothers, New Delhi, special senses, pp 219-220

7. Zur KB, Mu L, Sanders I (2004) Distribution pattern of the human lingual nerve. Clin Anat 17(2):88-92

8. Girod SC, Neukam FW, Girod B, Reumann K, Semrau H (1989) The fascicular structure of the lingual nerve and the chorda tympani: an anatomic study. J Oral Maxillofac Surg 47(6):607-609

9. Herzon FS, Martin AD (2006) Medical and surgical treatment of peritonsillar, retropharyngeal, and parapharyngeal abscesses. Curr Infect Dis Rep 8(3):196

10. Steyer TE (2002) Peritonsillar abscess: diagnosis and treatment. Am Fam Physician 65(1):93

Publisher's Note Springer Nature remains neutral with regard to jurisdictional claims in published maps and institutional affiliations. 